\title{
Structural Relations between Harmonic Sums up to $\mathrm{w}=6$
}

\author{
J. Blümlein ${ }^{1}$ and S. Klein ${ }^{1} *$
}

Deutsches Elektronen-Synchrotron, DESY, Platanenallee 6, D-15738 Zeuthen, Germany

\begin{abstract}
Multiply nested finite harmonic sums $S_{a_{1} \ldots a_{n}}(N)$ occur in many single scale higher order calculations in Quantum Field Theory. We discuss their algebraic and structural relations to weight $\mathbf{w}=6$. As an example, we consider the application of these relations to the soft and virtual corrections for Bhabha-scattering to $O\left(\alpha^{2}\right)$.
\end{abstract}

\section{Introduction}

Single scale processes in massless Quantum Field Theories $[2,3]$, or field theories being considered in the limit $m^{2} / Q^{2} \rightarrow 0$ [4], both for space- and time-like processes, exhibit significant simplifications when calculated in Mellin space if compared to representations in momentum-fraction $x$-space. Here, the Wilson coefficients and splitting functions are expressed by Nielsen-type integrals

$$
S_{n, p, q}(x)=\frac{(-1)^{n+p+q-1}}{\Gamma(n) p ! q !} \int_{0}^{1} \frac{d z}{z} \ln ^{(n-1)}(z) \ln ^{p}(1-z x) \ln ^{q}(1+z x)
$$

or harmonic polylogarithms [5]. The simplification is, to some extent, due to the structure of Feynman parameter integrals which possess a Mellin symmetry. The respective expressions can be expressed by finite harmonic sums $S_{a_{1} \ldots a_{n}}(N)$ for processes to 3-loop order [6,7], which form the appropriate language. Within the light-cone expansion, or analogous formalisms for time-like processes, the argument of the harmonic sums are even- or odd integers, depending on the process. However, one may consider mathematical generalizations, continuing the argument analytically to rational, real and complex values $N \in \mathbf{Q}, \mathbf{R}, \mathbf{C}$, respectively, $[6,8]$. In these extensions new relations between the harmonic sums are obtained, which lead to more compact representations. Since the hard-scattering cross sections usually have to be convoluted with non-perturbative parton densities, it is convenient to widely work in Mellin space using analytic representations, also for the solution of the evolution equations. This also applies to the treatment of heavy flavor contributions in the full phase space, for which concise semi-analytic representations were derived [9]. The final $x$-space results are obtained by a single numerical Mellin-inversion performed by a contour integral around the singularities of the problem.

In this note we give a summary on the algebraic and structural relations for finite harmonic sums, occurring in hard scattering processes. As an example we consider the virtual and soft QED corrections to Bhabha-scattering to $O\left(\alpha^{2}\right)$ in the on-mass-shell scheme [10] to show that also this process fits to the general basis-representation being derived for various other two- and three-loop QCD processes.

\footnotetext{
${ }^{*}$ This paper was supported in part by SFB-TR-9: Computergestütze Theoretische Teilchenphysik and Studienstiftung des Deutschen Volkes.
} 


\section{Algebraic Relations}

The complexity of finite harmonic sums is given by $N_{\max }=2 \cdot 3^{w-1}$, with the weight $w=\sum_{k=1}^{n}\left|a_{k}\right|$ growing exponentially. In the $x$-space representation of the 2 -loop Wilson coefficients in QCD, which are described by $w=4$, nearly 80 functions emerge, which corresponds to the maximum level possible, cf. [6,11]. The algebraic relations of finite harmonic sums operate on their index set and are implied by their quasi-shuffle algebra [12]. The algebraic relations of finite harmonic sums were investigated in [13] in detail. An example for the shuffle product is

$S_{a_{1}, a_{2}} \sqcup S_{a_{3}, a_{4}}=S_{a_{1}, a_{2}, a_{3}, a_{4}}+S_{a_{1}, a_{3}, a_{2}, a_{4}}+S_{a_{1}, a_{2}, a_{4}, a_{2}}+S_{a_{3}, a_{4}, a_{1}, a_{2}}+S_{a_{3}, a_{1}, a_{4}, a_{2}}+S_{a_{3}, a_{1}, a_{2}, a_{4}}$.

For a given index set the number of basic harmonic sums is counted by the number of Lyndon words and can be calculated by a Witt-formula. Investigating the type of harmonic sums emerging in physical single-scale problems up to 3 -loop order, cf. [2-4], the index $\{-1\}$ never occurs. Their number is $N_{\neg-1}=\left[(1-\sqrt{2})^{w}+(1+\sqrt{2})^{w}\right] / 2[14]$. One therefore may significantly reduce the number of basic functions using $N_{\neg-1}$ in the corresponding Witt formula. The following table illustrates the corresponding complexities of combinations \# $c$ and the number of algebraic basis elements $\#_{b}$ in dependence of the weight $w$. The initial complexity in case of the absence of indices $a_{l}=-1$ is lower than the number of algebraic basis elements in the complete case.

\begin{tabular}{|c|r|r|r|r|r|r|}
\hline$w$ & 1 & 2 & 3 & 4 & 5 & 6 \\
\hline$\#_{c}$ & 2 & 8 & 26 & 80 & 242 & 728 \\
$\#_{b}$ & 2 & 5 & 13 & 31 & 79 & 195 \\
\hline$\#_{c}(\neg-1)$ & 1 & 4 & 11 & 28 & 69 & 168 \\
$\#_{b}(\neg-1)$ & 1 & 3 & 7 & 14 & 30 & 60 \\
\hline
\end{tabular}

\section{Structural Relations}

The algebraic relations lead to a first reduction of the complexity of finite harmonic sums. They are independent of the value of these quantities. Beyond these relations, the structural relations are of a more specific type, cf. $[6,15]$. There are three types of these relations. The first class is implied by allowing half-integer values for $N$. A second class emerges through partial integration using the representations of harmonic sums through Mellin-transforms e.g. of weighted harmonic polylogarithms. The third set is implied by differentiating harmonic sums w.r.t. their argument, which requires $N \in \mathbf{R}$. To illustrate case 1 we represent

$$
\frac{1}{1-x^{2}}=\frac{1}{2}\left[\frac{1}{1-x}+\frac{1}{1+x}\right] \text {. }
$$

The Mellin transform of this equation implies, that $S_{-1}(N)$ is linearly dependent of $S_{1}(N)$, if $N \in \mathbf{Q}$. Various relations of this type emerge at higher weight [15]. If $N \in \mathbf{R}$, one may differentiate harmonic sums and obtains

$$
\frac{d}{d N} S_{a_{1} \ldots a_{n}}(N)=\int_{0}^{1} d x x^{N-1} \ln (x) f(x) .
$$

$S_{a_{1} \ldots a_{n}}(N)$ is given by the Mellin transform of $f(x)$. One may represent the derivative in terms of polynomials of harmonic sums and their values at $N \rightarrow \infty$. The analytic 
continuation of a harmonic sum to $N \in \mathbf{C}$ is a meromorphic function with poles at the nonpositive integers. Up to polynomials growing $\propto S_{1}^{m}(N),|N| \rightarrow \infty$, which can be separated, harmonic sums are factorial series. I.e. they obey an analytic recursion relation $F(z+1) \rightarrow$ $F(z)$ and one may calculate their asymptotic representation for $z \rightarrow \infty$ analytically. In this way these functions are uniquely defined in the complex plane. Due to the above properties we now define equivalence classes of representations, which contain a harmonic sum and all its derivatives. We will only count these equivalence classes, since all derivatives can be easily found analytically if the respective lowest weight sum of the class is known. In the following table we summarize the functions of the basic Mellin transforms up to $w=5$. The case $w=6$ is still to be completed.

$$
\begin{array}{llll}
w=1 & 1 /(x-1)_{+} & & \\
w=2 & \ln (1+x) /(x+1) & & \\
w=3 & \operatorname{Li}_{2}(x) /(x \pm 1) & & \\
w=4 & \operatorname{Li}_{3}(x) /(x+1) & S_{1,2}(x) /(x \pm 1) & S_{2,2}(x) /(x \pm 1) \\
w=5 & \operatorname{Li}_{4}(x) /(x \pm 1) & S_{1,3}(x) /(x+1) & \\
& \operatorname{Li}_{2}^{2}(x) /(x+1) & {\left[S_{2,2}(-x)-\operatorname{Li}_{2}^{2}(-x) / 2\right] /(x \pm 1)} & \\
w=6 & \operatorname{Li}_{5}(x) /(x+1) & S_{1,4}(x) /(x \pm 1) & \\
& S_{3,2}(x) /(x \pm 1) & \ldots &
\end{array}
$$

These functions emerge in the following processes : number of fct.

- $O(\alpha) \quad w \leq 2 \quad$ Wilson Coefficients/anom. dim. $\quad \# 1$

- $O\left(\alpha^{2}\right) w \leq 3 \quad$ Anomalous Dimensions \#2

- $O\left(\alpha^{2}\right) w \leq 4 \quad$ Wilson Coefficients $\quad \# \leq 5$

- $O\left(\alpha^{3}\right) w \leq 5 \quad$ Anomalous Dimensions \#15

- $O\left(\alpha^{3}\right) w \leq 6 \quad$ Wilson Coefficients $\quad \# 29+$

\section{Example: Bhabha Scattering}

As an example we consider the soft- and virtual corrections to Bhabha-scattering up to $O\left(\alpha^{2}\right), T_{2}(x)=\delta_{0}^{(2)}(x) \times\left(1-x+x^{2}\right) / x^{2},[10]$. The $x$-space expression simplifies in Mellin $N$ space and depends on three basic functions $S_{2,1,1}, S_{2,1}$ and $S_{1}$ only aside of polynomial pre-factors :

$$
\begin{aligned}
T_{2}(N)= & \frac{248+15 N^{2}+N^{4}}{2(N-2)(N-1) N(N+1)(N+2)} S_{1,1,1,1}(N)-\frac{2}{(N-1)(N+1)} \mathbf{S}_{\mathbf{2}, \mathbf{1}, \mathbf{1}}(N) \\
& +\frac{-340+120 N+17 N^{2}+18 N^{3}-31 N^{4}}{2(N-2)(N-1) N(N+1)(N+2)} S_{3,1}(N)-\frac{-304+278 N+81 N^{2}-38 N^{3}+19 N^{4}}{8(N-2)(N-1) N(N+1)(N+2)} S_{4}(N) \\
& +\frac{304-328 N-500 N^{2}+330 N^{3}-6 N^{4}+6 N^{5}-2 N^{6}+4 N^{7}}{(N-2)^{2}(N-1)^{2} N^{2}(N+1)(N+2)} \mathbf{S}_{\mathbf{2}, \mathbf{1}}(N) \\
& +\frac{-112-4 N^{2}-4 N^{4}}{(N-2)(N-1) N(N+1)(N+2)} S_{2,1}(N) \mathbf{S}_{\mathbf{1}}(N)+\frac{-48+8 N+6 N^{2}+7 N^{3}}{(N-1) N(N+1)(N+2)} S_{3}(N) S_{1}(N) \\
& +\frac{-1840+292 N+5532 N^{2}+827 N^{3}-1978 N^{4}-274 N^{5}+36 N^{6}+19 N^{7}-22 N^{8}}{4(N-2)^{2}(N-1)^{2} N^{2}(N+1)^{2}(N+2)} S_{1,1,1}(N)
\end{aligned}
$$




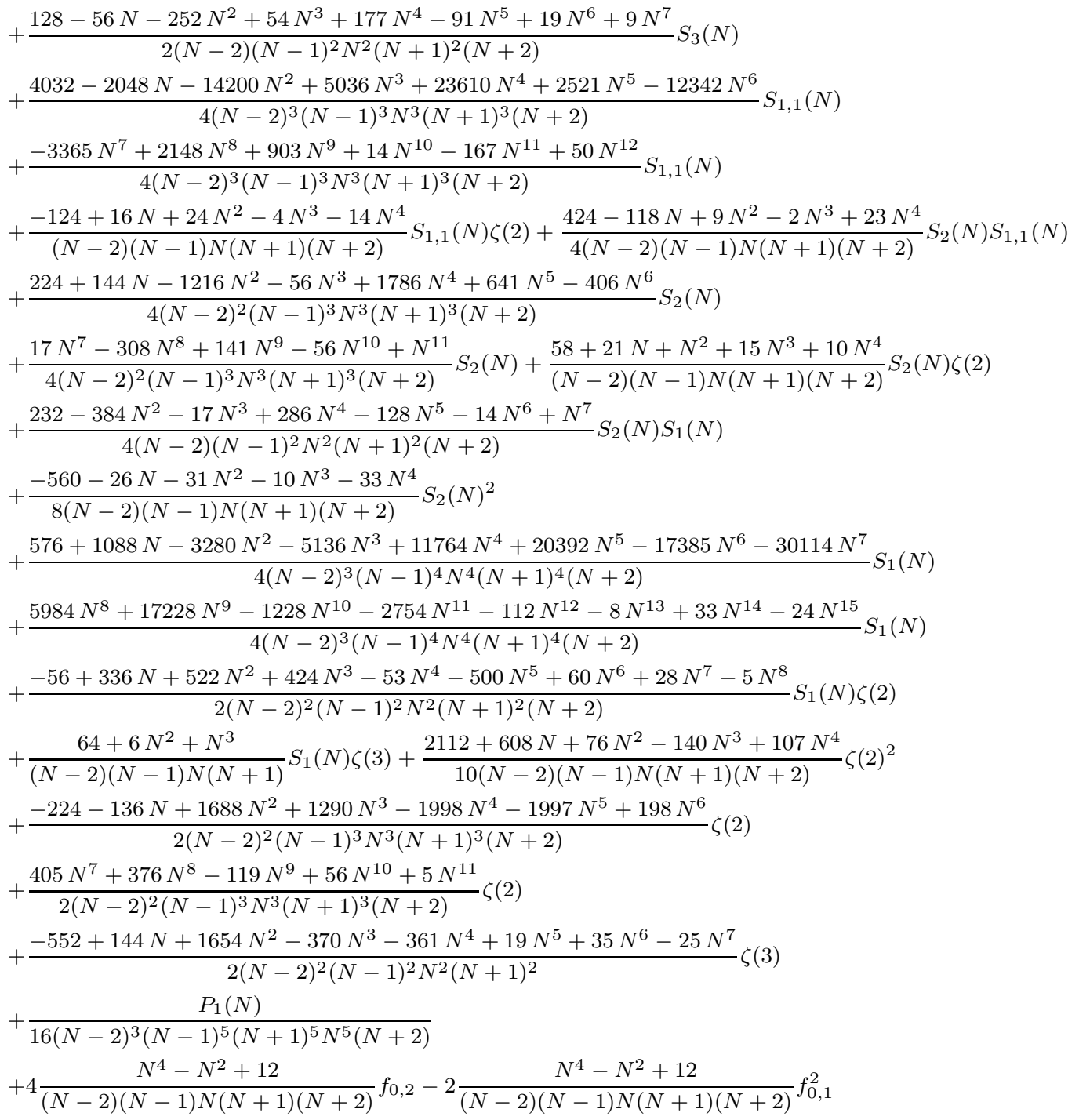

with

$$
\begin{aligned}
P_{1}= & 320-64 N-1920 N^{2}+1600 N^{3}+6524 N^{4}-14872 N^{5}-19036 N^{6}+31543 N^{7} \\
& -43960 N^{8}-13935 N^{9}+65372 N^{10}+26822 N^{11}-44576 N^{12}-9558 N^{13} \\
& +9840 N^{14}+339 N^{15}+428 N^{16}-371 N^{17}+128 N^{18}
\end{aligned}
$$

There are no alternating sums contributing, unlike the case for other 2-loop Wilson coefficients in QCD [2,4], where generally up to 6 functions emerge.

\section{Conclusions}

The single-scale quantities in Quantum Field Theories to 3 Loop Order, corresponding to $w=6$, can be represented in a polynomial ring spanned by a few Mellin transforms of the above basic functions, which are the same for all known processes, including QED 
processes, as the soft- and virtual corrections to Bhabha-scattering. This points to their general nature. The basic Mellin transforms are meromorphic functions with single poles at the non-positive integers. The total amount of harmonic sums reduces due to algebraic relations as a consequence of the index structure, and structural relations continuing the argument to $\mathrm{N} \epsilon \mathbf{Q}, \mathbf{N} \epsilon \mathbf{R}$. They can be represented in terms of factorial series up to simple "soft components". This allows an exact analytic continuation. Up to $w=6$ physical (pseudo-) observables are free of harmonic sums with index $=\{-1\}$. To $w=5$ all numerator functions are Nielsen integrals.

\section{References}

[1] Slides: http: //indico. cern. ch/contributionDisplay . py? contribId=212\&sessionId=6\& conf Id=9499

[2] J. Blümlein and V. Ravindran, Nucl. Phys. B 716 (2005) 128 [arXiv:hep-ph/0501178]; Nucl. Phys. B $\mathbf{7 4 9}(2006) 1$;

J. Blümlein and S. Moch, in preparation.

[3] A. Vogt, S. Moch and J. A. M. Vermaseren, Nucl. Phys. B 691 (2004) 129; 724 (2005) 3.

[4] J. Blümlein, A. De Freitas, W. L. van Neerven and S. Klein, Nucl. Phys. B 755 (2006) 272 ; I. Bierenbaum, J. Blümlein and S. Klein, arXiv:hep-ph/0703285; Phys. Lett. B 648 (2007) 195 ; J. Blümlein and S. Klein, DESY 07-027.

[5] E. Remiddi and J. A. M. Vermaseren, Int. J. Mod. Phys. A 15 (2000) 725.

[6] J. Blümlein and S. Kurth, Phys. Rev. D 60 (1999) 014018.

[7] J. A. M. Vermaseren, Int. J. Mod. Phys. A 14 (1999) 2037.

[8] J. Blümlein, Comput. Phys. Commun. 133 (2000) 76;

J. Blümlein and S. O. Moch, Phys. Lett. B 614 (2005) 53.

[9] S. I. Alekhin and J. Blümlein, Phys. Lett. B 594 (2004) 299.

[10] A. A. Penin, Phys. Rev. Lett. 95 (2005) 010408 ;

T. Becher and K. Melnikov, arXiv:0704.3582 [hep-ph];

S. Actis, M. Czakon, J. Gluza and T. Riemann, arXiv:0704.2400 [hep-ph] and Refs. therein.

[11] E. B. Zijlstra and W. L. van Neerven, Nucl. Phys. B 383 (1992) 525.

[12] M. E. Hoffman, J. Algebraic Combin. 11 (2000) 49.

[13] J. Blümlein, Comput. Phys. Commun. 159 (2004) 19.

[14] M. Dittmar et al., arXiv:hep-ph/0511119.

[15] J. Blümlein, Nucl. Phys. Proc. Suppl. 135 (2004) 225; DESY 07-042. 